\title{
O uso de portfólio na prática de atendimento domiciliar a idosos no curso de Medicina
}

\author{
The use of a portfolio in home health care \\ delivery to aged people during the medical course
}

\author{
Marcos Aparecido Sarria Cabrera ${ }^{1}$ \\ BarbaraTurini $^{1}$ \\ Lílian Bruniera Bruneli Paccola ${ }^{1}$
}

\author{
PALAVRAS-CHAVE \\ - Idoso. \\ - Assistência Domiciliar. \\ - Educação Médica. \\ - Geriatria.
}

Recebido em:30/06/2007 Reencaminhado em: 21/12/2007 Aprovado em: 07/02/2008

\begin{abstract}
R E S U M O
Este trabalho apresenta e analisa a utilização do portfólio na prática de atendimento domiciliar a idosos no Módulo do Envelhecimento do segundo ano do curso de Medicina da Universidade Estadual de Londrina. As informações analisadas foram obtidas dos 40 portfólios apresentados pelos alunos na conclusão do módulo em 2006. O portfólio é estruturado com dados de identificação, anamnese, exame físico, medicamentos, exames complementares e avaliações funcionais, cognitivas e sociais. As seguintes informações foram selecionadas para análise: descrição das visitas domiciliares e avaliação individual dos alunos. As principais vantagens identificadas pelos alunos foram à possibilidade de aplicação do conhecimento adquirido (50,0\%), a criação de vínculos $(37,2 \%)$ e o desenvolvimento da relação médico-paciente (35,9\%). Como principais dificuldades, apontaram insegurança (26,9\%), dúvida em relação ao consentimento $(15,4 \%)$ e invasão de privacidade $(12,8 \%)$. A análise sugere que a prática de atendimento domiciliar a idosos é importante para a formação de profissionais com condições de atender à população idosa. Além disso, o portfólio mostrou ser um recurso pedagógico que proporciona um aprendizado ativo e garante o envolvimento do aluno com o conteúdo proposto para a atividade.
\end{abstract}

\section{A B STRACT}

This study aims at presenting and analyzing the use of a portfolio in the home visits to aged people made by second year medical students attending the topic-based module "Aging" at the State University of Londrina. The information analyzed was collected from forty portfolios presented by the students on concluding the module in 2006. The portfolio contains data regarding identification of the patient, anamnesis, physical examination and complementary exams, medicines as well as functional, cognitive and social assessments. The information selected for analysis was: description and evaluation of the home visits by the students. The main advantages from the viewpoint of the students were the possibility of applying the acquired knowledge (50.0\%), the establishment of links (37.2\%) and the development of a good doctor-patient relationship (35.9\%). On the other hand, the main difficulties pointed out by the students were insecurity (26.9\%), doubt regarding consent (15.4\%) and invasion of privacy (12.8\%). The analysis suggests home health care delivery to be important in the education of future professionals capable of attending the aged population in a satisfactory way. Moreover, the portfolio showed to be a pedagogical tool contributing to active learning and making the students get involved with the content proposed for the activity.
REVISTA BRASILEIRA DE EDUCAÇ̃̃OMÉDICA

$18032(2): 180-187: 2008$

\footnotetext{
${ }^{1}$ Universidade Estadual de Londrina, Paraná, Brasil.
} 


\section{INTRODUÇÃO}

Nos últimos anos, vem crescendo a necessidade do ensino da Geriatria nas escolas médicas, visando à formação de profissionais com habilidades específicas para assistir à população ido$\mathrm{sa}^{1,2}$. Essas habilidades são desenvolvidas a partir de atividades pedagógicas específicas para essa faixa etária, como, por exemplo, a abordagem de pacientes de alta dependência, em instituições asilares e em domicílios ${ }^{3}$.

A Universidade Estadual de Londrina, onde desde 1998 foi implantado um currículo integrado de Medicina, com utilização de metodologias ativas de ensino-aprendizagem, oferta no segundo ano o módulo temático do Envelhecimento, com sete semanas de duração e que inclui entre as práticas desenvolvidas pelos estudantes o acompanhamento de uma pessoa idosa ${ }^{4}$. Durante os primeiros anos de organização do módulo, essa prática era proposta apenas em ambiente asilar, mas aos poucos foi também testada no cenário domiciliar e desde 2004 vem sendo desenvolvida apenas neste ambiente.

Entretanto, as atividades práticas desenvolvidas em ambientes não hospitalares apresentam dificuldades tanto na elaboração, quanto na supervisão e, principalmente, em sua avaliação ${ }^{5,6}$. A utilização de portfólios na avaliação é uma das propostas pedagógicas que possibilitariam uma reflexão acerca da atividade prática, bem como o desenvolvimento das estratégias de aprendizado ${ }^{7,8}$ e maior envolvimento do aluno na atividade proposta $^{9}$. O portfólio também possibilita um "diálogo reflexivo" entre professor e estudante na avaliação do processo de aprendizagem $^{10}$.

Este estuda apresenta e analisa a atividade prática de acompanhamento de idosos em seus domicílios realizada pelos estudantes ao longo das sete semanas de desenvolvimento do Módulo de Envelhecimento, a partir da análise dos portfólios por eles elaborados.

\section{METODOLOGIA}

\section{Material analisado}

Foram analisados os 40 portfólios elaborados em duplas de alunos do segundo ano do curso de Medicina da Universidade Estadual de Londrina durante as atividades da prática de atendimento domiciliar desenvolvida no Módulo de Envelhecimento, de setembro a novembro de 2006.

\section{Descrição do módulo temático do Envelhecimento - nível de graduação}

O módulo temático do Envelhecimento é elaborado por profissionais de diversas áreas da saúde, e seu desenvolvimento prevê atividades de grupo tutorial segundo a metodologia da aprendizagem por problemas, palestras e atividades práticas. Há diferentes modalidades de práticas, como atividades laboratoriais, avaliação nutricional, atividades clínicas hospitalares e atendimento domiciliar.

O objetivo geral do módulo é conhecer os diferentes estágios do processo de envelhecimento e suas patologias: da célula ao indivíduo na sociedade. Durante o desenvolvimento do módulo, espera-se que o aluno adquira conhecimentos a respeito das principais doenças crônico-degenerativas e suas terapêuticas, além dos determinantes psicossociais inerentes à população idosa ${ }^{11}$.

\section{Prática de atendimento domiciliar}

Essa prática propõe que os alunos (em duplas) acompanhem um idoso em seu domicílio, com visitas semanais durante o desenvolvimento do módulo. A cada ano, é escolhida uma Unidade Básica de Saúde do município, e os idosos acompanhados são selecionados pela coordenação de enfermagem da unidade, a partir da recomendação de que sejam idosos restritos ao domicílio e que estejam cadastrados pelo Programa da Saúde da Família local.

Os estudantes não contam com acompanhamento direto durante as visitas, ou seja, devem sozinhos encontrar o endereço indicado, apresentar-se e realizar as tarefas propostas sem o acompanhamento direto de um professor ou profissional de saúde. Também não há dias ou horários especificamente reservados para a realização das visitas. Os estudantes apenas devem, no final do módulo, relatar um mínimo de sete visitas realizadas ao longo das sete semanas de execução do módulo. Este fato permite grande flexibilidade para que cada dupla de estudantes combine os melhores momentos de visita com o seu idoso e os familiares.

\section{Portfólio}

As atividades desenvolvidas pelos alunos no domicílio são orientadas, referenciadas e avaliadas por meio de um portfólio a ser entregue no final das atividades do módulo (Quadro 1). 


\section{QUADRO 1}

Estrutura do portfólio

\begin{tabular}{|l|}
\hline 1. Fotografia do paciente junto com os alunos \\
\hline 2. Biografia do paciente (máximo de uma página) \\
\hline 3. Anamnese \\
\hline 4. Exame físico \\
\hline 5. Escala de dependência funcional (Katz) \\
\hline 6. Avaliação nutricional (miniavaliação nutricional - MAN) \\
\hline 7. Avaliação cognitiva (miniexame do estado mental) \\
\hline 8. Escala de depressão geriátrica \\
\hline 9. Farmacocinética e farmacodinâmica dos medicamentos utilizados \\
\hline 10. Exames complementares disponíveis \\
\hline 11. Diagnóstico social \\
\hline 12. Comentários do paciente e/ou cuidadores \\
\hline 13. Comentários dos alunos a respeito da prática (vantagens e dificuldades) \\
\hline
\end{tabular}

Os problemas estudados durante as atividades tutoriais ao longo do módulo levam os alunos a conhecer certos instrumentos utilizados para a avaliação de determinadas condições clínicas das pessoas idosas, como, por exemplo, as escalas de referência para avaliações do grau de dependência, nível cognitivo ou grau de depressão em pessoas de idade avançada. Com as práticas propostas no atendimento domiciliar, os estudantes têm a oportunidade de aplicar esses instrumentos na pessoa que estão acompanhando, à medida que vão tomando conhecimento dos mesmos.

Os portfólios são analisados pelo docente responsável pela prática, sendo avaliados os seguintes itens: apresentação geral do trabalho, requisitos mínimos solicitados, aspectos relacionados à reflexão do aluno, auto-avaliação e análise qualitativa do conteúdo apresentado.

\section{Metodologia de coleta dos dados}

Os dados foram obtidos a partir da análise quantitativa e qualitativa dos 40 portfólios apresentados no término do Módulo de Envelhecimento no ano de 2006. Cada portfólio contém os dados do idoso atendido e as avaliações dos dois alunos. As seguintes variáveis foram analisadas: idade e sexo dos idosos atendidos e avaliações dos alunos sobre a prática.

No final do módulo, foi solicitado que cada aluno apresentasse por escrito uma avaliação dos pontos positivos, negativos e sugestões referentes à prática de atendimento domiciliar.
Foram realizadas análises qualitativas das informações apresentadas pelos alunos, individualmente, em relação a vantagens e dificuldades encontradas. Inicialmente, foi registrado todo o conteúdo apresentado nos dois itens (vantagens e dificuldades). Em seguida, estes dados foram interpretados e categorizados para análise. Do total de 80 alunos, dois estudantes não apresentaram a avaliação da prática $(2,5 \%)$.

Além dessas análises, foram selecionados aleatoriamente quatro portfólios para exemplificação do cronograma de visitas realizadas pelos alunos.

\section{RESULTADOS}

Foram analisados os 40 portfólios apresentados pelas duplas de estudantes como relatório final da prática de atendimento domiciliar, em que foram descritas as atividades desenvolvidas durante cada uma das visitas.

Em relação ao perfil dos idosos envolvidos nas atividades, a idade variou de 64 a 95 anos (média = 78,2 anos), com predomínio do sexo feminino - $29(72,5 \%)$.

A descrição individualizada de algumas visitas domiciliares realizadas pelos alunos demonstrou que houve flexibilidade das atividades de acordo com o idoso e a realidade encontrada, mantendo como referência a proposta de estrutura do portfólio a ser elaborado (Quadro 2). 


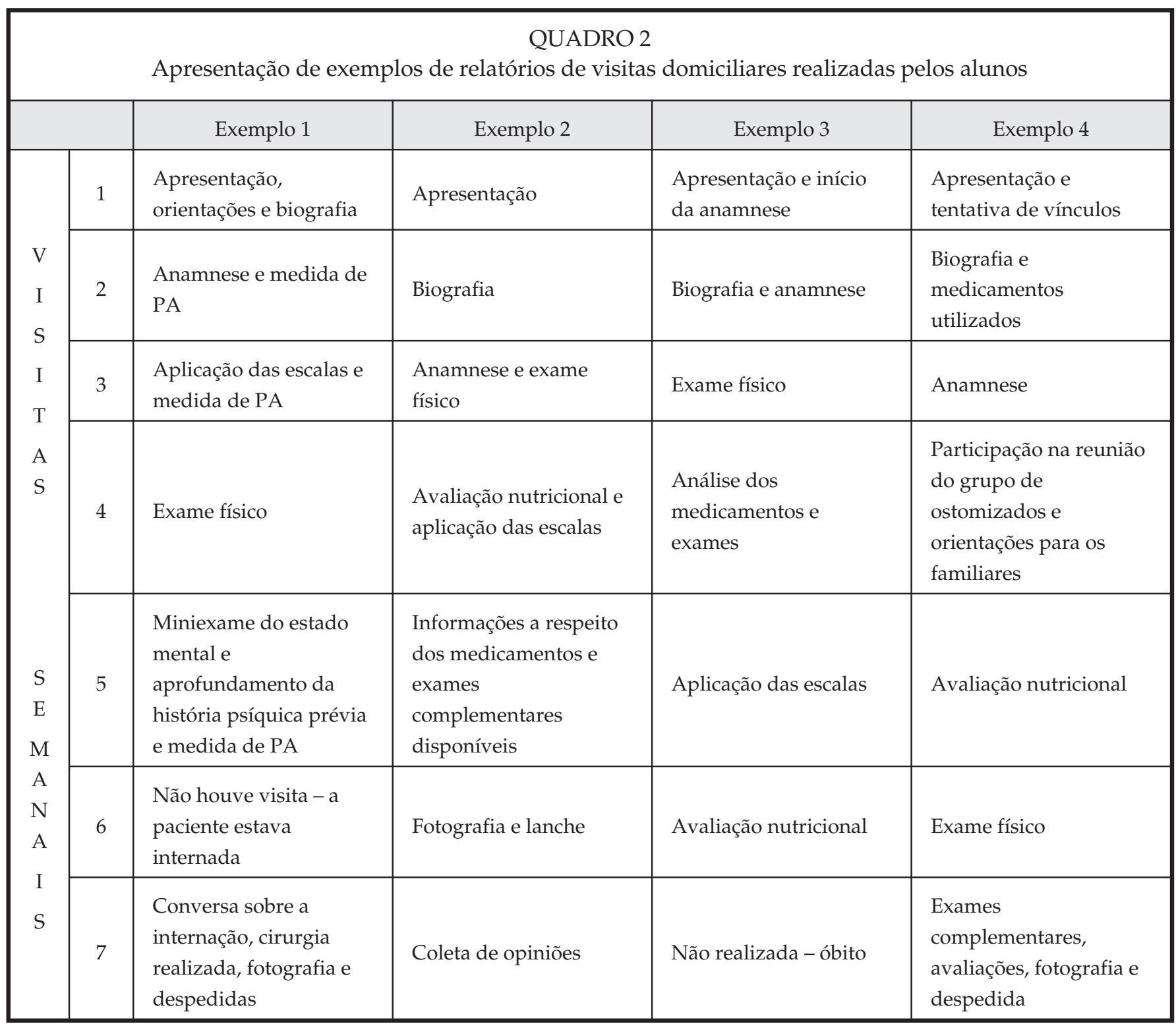


Na análise das avaliações dos alunos a respeito da prática de atendimento domiciliar, os dados contidos nos portfólios identificaram a possibilidade de aplicar os conhecimentos e o estímulo ao estudo como a principal vantagem da atividade (50,0\%). Os alunos também apontaram como outras vantagens importantes à criação de vínculo $(37,2 \%)$, melhora da relação médico-paciente $(35,9 \%)$, recompensa afetiva da relação com os idosos $(29,5 \%)$ e percepção diferenciada dos valores da pessoa idosa $(21,8 \%)$. Alguns aspectos relacionados à facilitação de atividades práticas no domicílio foram citados, bem como o contato com a realidade encontrada na comunidade. Temas mais complexos também foram contemplados pelos alunos, como o estímulo à vocação para a Medicina, a reflexão a respeito da morte e a análise da situação na perspectiva do cuidador (Tabela 1).

TABELA 1

Vantagens da prática domiciliar segundo os alunos

\begin{tabular}{lll}
\hline Vantagens & $\mathrm{n}(78)^{*}$ & $\%$ \\
\hline Aplicação de conhecimentos e estímulo para estudar & 39 & 50,0 \\
Possibilidade de criação de vínculos & 29 & 37,2 \\
Melhora da relação médico-paciente & 28 & 35,9 \\
Recompensa afetiva & 23 & 29,5 \\
Maior contato com a realidade & 19 & 24,4 \\
Possibilidade de atividades práticas & 19 & 21,8 \\
Percepção dos valores dos idosos & 17 & 19,2 \\
Treinamento em assistência no domicilio & 15 & 19,2 \\
Entendimento global da doença & 15 & 19,2 \\
Contribuição para os problemas dos idosos & 15 & 12,8 \\
Maior estímulo à vocação para a Medicina & 10 & 7,7 \\
Conhecimento da situação do cuidador & 6 & 5,1 \\
\hline
\end{tabular}

* Dois alunos não responderam. 
Os alunos apontaram a insegurança como a maior dificuldade apresentada durante o desenvolvimento da prática (26,9\%). As dúvidas em relação ao consentimento do paciente e dos cuidadores e a possibilidade de invasão da privacidade da família foram outros problemas identificados na avaliação dos alunos. Outros problemas mencionados foram as dificuldades de comunicação com o paciente, a abordagem das incapacidades, o baixo interesse do idoso e dificuldade em formar vínculos (Tabela 2).

TABELA 2

Dificuldades identificadas durante a prática domiciliar segundo os alunos

\begin{tabular}{lcc}
\hline Dificuldades & $\mathrm{n}(78)^{*}$ & $\%$ \\
\hline Insegurança & 21 & 26,9 \\
Dúvida em relação ao consentimento do paciente & 12 & 15,4 \\
Receio em invadir a privacidade & 10 & 12,8 \\
Dificuldade de comunicação com o idoso & 10 & 12,8 \\
Dificuldade em relação a idoso com incapacidades & 6 & 7,7 \\
Baixo interesse do idoso & 4 & 5,1 \\
Dificuldades de criação de vínculos & 3 & 3,8 \\
Falta de relacionamento com a Unidade Básica de Saúde & 2 & 2,6 \\
Rompimento do vínculo adquirido & 2 & 2,6 \\
Frustração com o resultado obtido & 1 & 2,6 \\
Dificuldade em resolver os problemas encontrados & 1,3 \\
Dificuldade de aplicar as escalas & 1 & 1,3 \\
Diferenças em relação à idade e ao nível social & 1 & 1,3 \\
\hline Falta de tempo disponível para a prática & 1 & 2 \\
\hline
\end{tabular}

* Dois alunos não responderam. 


\section{DISCUSSÃO E CONCLUSÕES}

Os dados apresentados mostram que a proposição do portfólio na avaliação de uma prática de atendimento domiciliar com idosos é uma estratégia que proporciona a realização de atividades coerentes com o conteúdo proposto e possibilita a avaliação mais profunda dos alunos e do processo de ensino e aprendizado.

Um dos desafios das escolas médicas, atualmente, é preparar os alunos para o atendimento ao idoso, respeitando suas características e peculiaridades ${ }^{12,13}$. Neste sentido, a análise das atividades apresentadas evidencia que a prática de atendimento domiciliar pode contribuir para o desenvolvimento destas habilidades diante do paciente idoso, pois tem uma estrutura de acompanhamento longitudinal dos pacientes e um processo de aprendizado ativo e centrado no aluno.

O ensino centrado no aluno tem sido proposto por muitos autores como uma forma eficaz de formação de profissionais. No Brasil, as diretrizes curriculares para os cursos de graduação em Medicina recomendam especificamente, em seu artigo 12, a utilização de "metodologias que privilegiem a participação ativa do aluno na construção do conhecimento e a integração entre os conteúdos". O mesmo artigo também sugere a inserção precoce do aluno em "atividades práticas relevantes para a sua futura vida profissional", bem como a utilização de "diferentes cenários de ensino-aprendizagem, permitindo ao aluno conhecer e vivenciar situações variadas de vida, da organização da prática e do trabalho em equipe multiprofissional" e a "interação ativa do aluno com usuários e profissionais de saúde"14.

Deve-se ressaltar o papel da Unidade Básica de Saúde local no desenvolvimento das atividades, pois, além de servir como referência territorial, foram os profissionais da unidade que escolheram os idosos a serem visitados. Esta integração com os serviços vai ao encontro da necessidade de diversificar os cenários para a formação dos profissionais de saúde $\mathrm{e}^{15} \mathrm{e}$ aproxima os alunos da realidade do atendimento ao idoso em nível primário.

A estruturação do portfólio com base nas habilidades a serem desenvolvidas durante o curso foi muito bem avaliada pelos alunos e contribuiu para sedimentar o conteúdo teórico do módulo. Tigelaar et al. ${ }^{16}$ analisaram a impressão dos professores de Medicina envolvidos com a utilização de portfólios e observaram, como uma das vantagens, a possibilidade de flexibilização e ajuste às necessidades individuais.

Além de representar uma ótima maneira de aplicar os conhecimentos adquiridos, a análise das vantagens identificadas pelos alunos aponta outras características favoráveis à prática domiciliar proposta, entre elas a possibilidade de maior relacionamento com o paciente e a família, e um olhar para aspectos do paciente e do cuidador que dificilmente seriam atingidos em práticas em ambiente hospitalar. A inclusão da fotografia e da biografia como itens obrigatórios do portfólio pode ter contribuído para essa boa interação. Outros autores também mostraram que o desenvolvimento de habilidades reflexivas é facilitado com o uso de portfólios ${ }^{17,18}$.

A sugestão, por um grupo de alunos, de que a prática fortaleceu os sentimentos em relação à vocação profissional é um dado extremamente relevante, pois, tanto nas escolas médicas quanto no conjunto da sociedade, encontramos movimentos que dificultam o fortalecimento desses sentimentos.

Em suas avaliações, os alunos identificaram outros temas complexos, que representam alguns desafios na formação gerontológica dos profissionais da área da saúde, como o entendimento e o amadurecimento diante da finitude e da morte ${ }^{19}$.

Contudo, apesar de reconhecermos o grande potencial pedagógico destas atividades, algumas observações dos alunos devem ser consideradas, como a insegurança, o receio do não consentimento e da invasão da privacidade. Uma das alternativas que minimizariam estas dificuldades seria a adoção de uma estrutura de supervisão docente mais próxima da unidade familiar. Neste sentido, para a proposição destas atividades é necessária uma preparação maior do aluno e da família, mas que não comprometa a independência necessária e positiva desta inter-relação (aluno-paciente-família).

Nossos dados chamam a atenção para as vantagens da criação de práticas de atendimento domiciliar com esta população geriátrica. Além disso, sugerem que o portfólio representa um instrumento fundamental para atingir os objetivos pedagógicos propostos.

Assim, esperamos contribuir para a promoção da saúde dos idosos com a formação de profissionais não só mais informados, como também mais amadurecidos e sensibilizados diante do envelhecimento.

\section{REFERÊNCIAS}

1. Garcez-Leme LE, Leme MD, Espino DV. Geriatrics in Brazil: a big country with big opportunities. J Am Geriatr Soc. 2005; 53(11): 2018-22.

2. Kinsella KG. Future longevity-demographic concerns and consequences. J Am Geriatr Soc. 2005; 53(Suppl 9):S299-S303.

3. American Geriatrics Society Core Writing Group of the Task Force on the Future of Geriatric. Caring for older Americans: The Future of Geriatric Medicine. J Am Geriatr Soc. 2005; 53(Suppl 6):S245-S256. 
4. Cabrera MAS, Almeida JR, Turini B, Martin G, Domiciano SP. Ensino da geriatria através do aprendizado baseado em problemas - experiência da Universidade estadual de Londrina, PR. Gerontologia. 2000; 8(2):56-62.

5. Feurwerker L, Costa H, Rangel ML. Diversificação de cenários de ensino e trabalho sobre necessidades / problemas da comunidade. Divulg Saúde Debat. 2000; 22: 36-48.

6. Scott-Tilley D, Marshall-Gray P, Valadez A, Green A Integrating long-term care concepts into Baccalaureate Nursing Education: The road to geriatric health care. J Nurs Educ. 2005; 44(6): 286-290.

7. Seiffert OMLB. Portfólio de avaliação do aluno: como desenvolvê-lo?. Olho Mágico.[online] 2001; 8(2) [capturado 25 abr. 2007]. Disponível em: http://www.ccs.uel.br/olhomagico/v8n1/index.html

8. Alvarenga, GM. Portfólio: o que é e a que serve? Olho Mágico. [online] 2001; 8(2) [capturado 25 abr. 2007]. Disponível em: http://www.ccs.uel.br/olhomagico/v8n1/index.html.

9. Webb TP, Aprahamian C, Weigelt JA, Brassel KJ. The surgical learning and instructional portfolio as a self-assessment educational tool demonstrating practice-based learning. Curr Surg. 2006; 63(6): 444-7.

10. Alves LP. Portfólios como instrumentos de avaliação dos processos de ensinagem. In: Anastasiou LGC, Alves LP, org. Processoso de Ensinagem na Universidade. Pressupostos para estratégias de trabalho em aula. 5. ed. Joinville, SC: Ed. Univille; 2005. p.101-20.

11. Cabrera MAS, Paccola LBB. Processo de envelhecimento: 3MOD213. Londrina: Ed.UEL; 2006. 35 p.

12. Motta LB. Formando médicos para o desafio do envelhecimento com qualidade no Brasil: uma contribuição à elaboração de currículos. Rio de Janeiro; 2004. Mestrado [Dissertação] - Universidade do Estado do Rio de Janeiro Instituto de Medicina Social.
13. Keller I, Makipaa A, Kalenscher T, Kalache A. Global Survey on Geriatrics in the Medical Curriculum. Geneva: World health organization; 2002.

14. Brasil. Ministério da Educação. Conselho Nacional de Educação. Câmara de Educação Superior. Resolução CNE/CES no 4 de 7 de novembro de 2001. Institui Diretrizes Curriculares Nacionais do Curso de Graduação em Medicina. Diário Oficial da União. Brasília, 9 nov. 2001; Seção 1, p.38.

15. Cabrera MAS. Integração educação -assistência. In: Freitas E. Tratado de Geriatria e Gerontologia. Rio de Janeiro: Guanabara Koogan; 2006. p.1490-4.

16. Tigelaar DE, Dolmans DH, De grave WS, Wolfhagen IH, Van der Vleuten CP. Participants' opinions on the usefulness of a teaching portfolio. Med Educ. 2006; 40(4): 371-8.

17. Pitkala KH, Mantyranta T. Feelings related to first patient experiences in medical school. A qualitative study on students' personal portfolios. Patient Educ Couns. 2004; 54(2): 171-7.

18. Driessen EW, Van Tartwijk J, Overeem K, Vermunt JD, Van der Vleuten CP. Conditions for successful reflective use of portfolios in undergraduate medical education. Med Educ. 2005; 39(12): 1230-5.

19. Py L , org. Finitude: uma proposta para reflexão e prática em Gerontologia. Rio de Janeiro: NAU; 1999. 192 p.

\section{CONFLITOS DE INTERESSE}

Declarou não haver

\section{ENDEREÇO PARA CORRESPONDÊNCIA}

Marcos Aparecido Sarria Cabrera

Rua Montese, 65A

86015-020 - Londrina - PR

e-mail: marcoscabrera@uol.com.br 